\title{
Variations in the Innervation of the Long Head of the Triceps Brachii: A Cadaveric Investigation
}

\author{
Alexandra J. Erhardt DO, Bennett Futterman MD
}

Received: 9 May 2016/Accepted: 28 September 2016/Published online: 9 November 2016

(C) The Association of Bone and Joint Surgeons \& 2016

\begin{abstract}
Background Some leading anatomy texts state that all three heads of the triceps brachii are innervated by the radial nerve. The posterior cord of the brachial plexus bifurcates to terminate as the radial and axillary nerves. Studies have noted the presence of axillary innervation to the long head of the triceps brachii muscle, patterns different from the classic exclusive radial nerve supply. An understanding of these variations may assist the clinician in the assessment of shoulder weakness and in preoperative and operative planning of radial and axillary neuropathies. Questions/Purpose We aimed to further investigate, in cadaver dissections, the prevalence of axillary nerve contribution to the innervation of the long head of the triceps brachii.

Methods We performed bilateral brachial plexus dissections on 10 embalmed cadavers combining anterior axillary

Each author certifies that he or she has no commercial associations (eg, consultancies, stock ownership, equity interest, patent/licensing arrangements, etc) that might pose a conflict of interest in connection with the submitted article.

All ICMJE Conflict of Interest Forms for authors and Clinical Orthopaedics and Related Research ${ }^{\circledR}$ editors and board members are on file with the publication and can be viewed on request.

Each author certifies that his or her institution approved or waived approval for the human protocol for this investigation and that all investigations were conducted in conformity with ethical principles of research.
\end{abstract}

\section{A. J. Erhardt ( $\square)$}

Department of Surgery, Hackensack UMC Palisades, 7600 North

River Road, North Bergen, NJ 07047, USA

e-mail: erhardt.alexandra@gmail.com

\section{B. Futterman}

Department of Anatomy, New York Institute of Technology

College of Osteopathic Medicine, Old Westbury, NY, USA and posterior subscapular approaches. Two additional unilateral cadaveric brachial plexuses were dissected. The posterior cords were fully dissected from the roots distally. The radial and axillary nerves were followed to their muscle insertion points, the dissections were photographed, and the length of branching segments were measured.

Results Of the 10 paired cadavers dissected (20 specimens), in only one of the 10 cadavers was the classic innervation pattern of radial nerve observed. The other nine cadavers had varying patterns of radial and axillary nerve innervation, The observed patterns were radial and axillary (dual) on one side with radial alone on the other, dual innervation bilaterally, or axillary with contralateral radial innervation. The two additional unilateral dissected specimens were innervated exclusively by the axillary nerve.

Conclusions Gross and surgical anatomy sources state that the radial nerve is the sole nerve supply to the long head of the triceps. In our study sample, pure radial innervation of the long head of the triceps brachii was not the predominant nerve pattern. We found four other studies that looked at axillary innervation of the long head of the triceps; of the 62 total cadaver shoulders examined in those studies, $71 \%$ were found to have nonclassic innervation patterns. Nonclassic patterns may include purely axillary, dual, or posterior cord innervation to the long head of the triceps, and may account for the majority of innervation to the long head of the triceps. These are similar to our findings.

Clinical relevance Understanding the innervation of the long head of the triceps and variations in axillary nerve course is critical to the clinical diagnosis of injury, surgical treatment options, and rehabilitation of axillary nerve injuries. With this information, the practitioner may have additional surgical options, clearer rationales for clinical situations, and explanations for patient outcomes. 


\section{Introduction}

Some leading anatomy textbooks describe the innervation to all three heads of the triceps brachii muscle as from the radial nerve $[8,11]$. The radial nerve originates from the posterior cord of the brachial plexus at the bifurcation with the axillary nerve. The radial nerve carries nerve fibers from the $\mathrm{C} 5$ to $\mathrm{T} 1$ nerve roots, whereas the axillary is said to carry predominantly $\mathrm{C} 5$ and $\mathrm{C} 6$ nerve root fibers. The axillary nerve is known to be the motor supply to the teres minor and deltoid muscles. The teres minor, originating from the scapula and inserting to the humerus, forms the superior border of the quadrangular space - an anatomic landmark and space implicated in some shoulder dysfunction syndromes [10, 13]. The edge of the humerus forms the lateral border of the quadrilateral space, the teres major muscle forms the inferior border, and the long head of the triceps forms the medial border. The quadrangular space is used as a landmark to identify the posterior circumflex humeral artery and the axillary nerve after they exit the axilla [11]. After traversing the quadrangular space, the axillary nerve may split into anterior and posterior branches, if it has not already done so. From these branches the axillary nerve will innervate the teres minor, deltoid, and inferior aspect of the glenohumeral joint capsule. The exact course of the axillary nerve after exiting the quadrilateral space has proven to be quite variable $[1,3,6,7,15]$.

Although the textbooks do not describe an innervation to the long head of the triceps other than the radial nerve, some cadaveric studies have noted cases of the motor branch of the long head of the triceps arising from the axillary nerve $[5,12,14]$. Clinical scenarios of axillary nerve injury with unexpected triceps brachii dysfunction support a cadaver study documenting this variant [14].

We aimed to further investigate the prevalence of axillary nerve innervation of the long head of the triceps brachii muscle and to elaborate on the specific innervation patterns.

\section{Materials and Methods}

Twenty-two brachial plexuses of 12 embalmed cadavers (six male, six female) were dissected, none of which was observed to have evidence of prior shoulder injury. Two of the cadavers had unilateral dissections, whereas 10 had bilateral brachial plexus dissections accounting for a total of 22 plexus specimens. The mean age of the cadavers was 80 years (range, 59-94 years). All cadavers were white and from the upstate region of New York. These cadavers were among the cadavers used in our medical school gross anatomy program. They were chosen from a larger pool of specimens because they had not had previous deep shoulder region dissections.

Dissection was performed through anterior and modified posterior subscapular approaches that allowed for full exposure of the posterior cord of the brachial plexus and quadrangular space containing the exiting axillary nerve [9].

\section{Anterior Approach}

A deltopectoral incision was made from the clavicle to the axilla. Skin and subcutaneous tissue were removed to observe the muscles and vessels. The humeral attachment of the pectoralis muscle was cut and reflected medially. Careful dissection of the adipose tissue surrounding the neurovasculature of the axilla was done to observe the divisions and cords of the brachial plexus. After proper identification of the posterior cord and subsequently the axillary and radial nerves, the remainder of the dissection was performed through a subscapular approach.

\section{Posterior Subscapular Approach}

After skin and subcutaneous tissue were reflected to reveal the underlying musculature, the posterior deltoid was released from the attachment to the scapular spine and then reflected laterally. Through careful dissection and removal of adipose tissue, the quadrangular space was identified by the borders of the teres minor muscle superiorly, humerus laterally, teres major inferiorly, and long head of the triceps medially. Exiting the quadrangular space, the axillary nerve was readily identifiable. Under $\times 2$-loupe magnification, the borders of the proximal aspect of the long head of the triceps were identified and examined closely for any entering neurovascular structures. The axillary nerve was carefully dissected from its origination off the posterior cord to its branches inserting in the teres minor, deltoid, and in many cases the long head of the triceps brachii muscles.

Photographs were taken of all specimens (Fig. 1). Quantitative data of the branching pattern were collected and nerve maps created. The nerve map was a drawing created from a photograph of the dissection and caliper measurements taken of the length of nerve segments from branch points. When axillary innervation to the long head of the triceps was observed, samples of the nerve, at the point of neuromuscular insertion, were taken for hematoxylin and eosin histologic examination and confirmation of the presence of nerve tissue. In our presentation of the data, we denote exclusive radial nerve innervation to the long head of the triceps as the classic pattern and any 


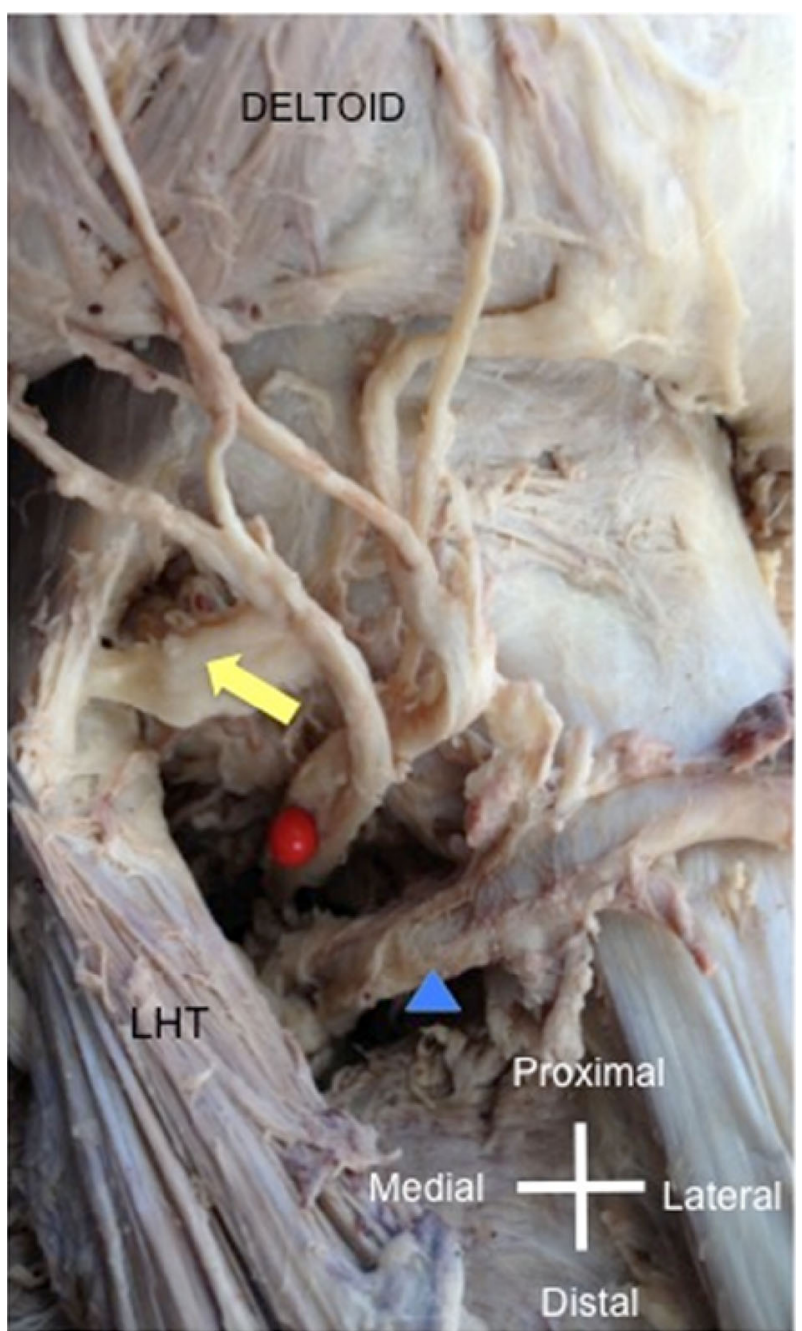

Fig. 1 A posterior view of a right shoulder dissection is shown. LHT $=$ long head of triceps muscle; red dot $=$ axillary nerve, posterior branch; yellow arrow $=$ nerve to long head of the triceps, blue arrowhead $=$ posterior circumflex humeral artery.

variation from this pattern as nonclassic. Therefore, nonclassic innervation consists of any innervation pattern other than uniquely radial nerve contribution (ie, branch from axillary nerve-only or dual innervation from axillary and radial nerves).

\section{Results}

Eight of the 22 shoulders dissected were observed to have exclusive innervation by the radial nerve to the long head of the triceps, the classic innervation pattern. Fourteen of the 22 specimens however, had nerve patterns other than the classic pattern. Of the nonclassic patterns, 11 of the 22 revealed dual innervation (axillary and radial) and three had axillary supply only. None of the dissections showed innervation to the long head of the triceps from the posterior cord alone.

When viewing the data with the two unilateral dissections removed, that is, the bilateral analysis from 10 cadavers, we found that three of the 10 had dual innervation bilaterally, five of the 10 had dual innervation on one side and radial innervation on the other, one of 10 had radial on one side and axillary on the opposite side, and only one cadaver had bilateral radial innervation.

\section{Discussion}

Anatomic sources have stated that the radial nerve innervates all three heads of the triceps brachii muscle $[8,11]$. The findings of some studies challenge the traditional notions of the classic pattern $[5,12,14]$. Cases of axillary nerve innervation to the long head of the triceps have been documented in cadaveric specimen in several studies $[4,5,12]$. Our purpose for this study was to provide innervation data for the long head of the triceps regarding additional brachial plexuses and to determine if our sample would result in findings similar to those in the published series.

Our study had several limitations. Our sample size was small, however, a pattern of innervation seemed to be present. The long head of the triceps brachii often is supplied to some degree by branches of the axillary nerve. Additionally, we did not attempt to determine if the nerve bundles had motor, sensory, or mixed functionality. Our specimens may not be representative of all populations as they were from a geographic region distinctly different from those in the prior publishes studies $[4,5,12]$. The actual ancestry data of the individual cadavers were not available.

We have expanded the existing data base regarding innervation of the long head of the triceps of these studies by dissecting 22 shoulders ( 12 cadavers). We found variations in the innervation patterns of the long head of the triceps [8, 11]. Our study confirms previous findings $[5,12,14]$ of axillary contribution to the long head of the triceps. We observed that 12 of the 22 cadaver shoulders have at least some degree of axillary nerve supply to the long head of the triceps. In three cases the innervation to the long head of the triceps was exclusively from the axillary nerve and 11 of the 22 revealed dual innervationaxillary and radial. The classic innervation pattern, exclusive supply by the radial nerve, was observed in the minority of cases. Of the nonclassic patterns, our results provide additional data clarifying the presence of this dual innervation (radial and axillary) in half of the specimens. Other studies suggest the nonclassic innervation pattern to the long head of the triceps was present in more than half 
of the cases. Forty-four of the 62 total cadaver shoulders examined by various research groups $[2,5,12,14]$ showed innervation patterns other than exclusive radial. Aszmann et al. [2] described the distribution and variability of innervation of the shoulder and noted a small branch arising from the axillary nerve inserted in the tendinous portion of the triceps in seven of 25 cadaver specimens. In a case report by Nanjundaiah et al. [12], one cadaver was found to have the nerve to the long head of the triceps originate from the axillary nerve, at its bifurcation in the anterior and posterior branches. They reported this to be bilaterally symmetric, although it was unclear whether the long head of the triceps also had a branch from the radial nerve. de Sèze et al. [5] dissected 20 posterior cords from 10 cadaveric specimens to delineate the origin of the motor branch to the long head of the triceps. They found that in $65 \%$ of cases the motor branch to the long head of the triceps originated from the axillary nerve, and the remaining arose from a branch of the posterior cord, and in no case from the radial nerve. Rezzouk et al. [14] dissected 15 posterior cords and found no case where the long head of the triceps received innervation from the radial nerve. Our study confirms the findings of previous studies $[5,12,14]$ that the classic radial innervation pattern is less common than the nonclassic innervation patterns.

Our findings differ from those of other series in that we found that the long head of the triceps brachii was supplied by branches from the axillary and radial nerves. This pattern was not reported in the other series $[2,5,12,14]$ and we found this pattern in half of the specimens. We also found that the radial nerve is at times the exclusive innervation of the long head of the triceps.

Innervation of the long head of triceps brachii appears to be quite variable and complex carrying with it relevance to shoulder injury and current approaches to surgical nerve repair. Awareness of the possible dual innervation of the long head of the triceps has potential value in the surgical treatment of deltoid dysfunction attributable to axillary nerve injury [4]. In an individual with dual innervation, the axillary branch to the triceps could be rerouted to the deltoid while the radial nerve branch to the triceps could be left intact to maintain triceps function. Based on our study, this pattern would be available in a large number of patients. Clinical cases of isolated axillary nerve damage with an unexpected decrease in triceps extensor function have been reported [14]. This is supported by the finding of exclusive axillary nerve supply to the long head of the triceps. In a surgical approach to the triceps brachii, the nerve branch to the muscle would be found more proximally than the expected location of a radial nerve branch in an individual with axillary innervation.

Our investigation of innervation of the long head of the triceps reveals that a more-complex pattern of dual innervation of the long head of the triceps may commonly occur. Our study also confirms previous findings $[2,5,12,14]$ of axillary contribution to long head of the triceps. An understanding of these variations may influence the surgical approach and reconstructive options available in neuromuscular reconstructive practice. Further investigations are needed to determine whether both branches are of motor, sensory, or mixed function in the dual-innervated cases and the amount of function each branch in the dualsupplied muscle provides.

\section{References}

1. Apaydin N, Tubbs RS, Loukas M, Duparc F. Review of the surgical anatomy of the axillary nerve and the anatomic basis of its iatrogenic and traumatic injury. Surg Radiol Anat. 2010;32:193-201.

2. Aszmann OC, Dellon AL, Birely BT, McFarland EG. Innervation of the human shoulder joint and its implications for surgery. Clin Orthop Relat Res. 1996;330: 202-207.

3. Ball CM, Steger T, Galatz LM, Yamaguchi K. The posterior branch of the axillary nerve: an anatomic study. J Bone Joint Surg Am. 2003;85:1497-1501.

4. Bertelli JA, Kechele PR, Santos MA, Duarte H, Ghizoni MF. Axillary nerve repair by triceps motor branch transfer through an axillary access: anatomical basis and clinical results. J Neurosurg. 2007;107:370-377.

5. de Sèze MP, Rezzouk J, de Sèze M, Uzel M, Lavignolle B, Midy D, Durandeau A. Does the motor branch of the long head of the triceps brachii arise from the radial nerve? An anatomic and electromyographic study. Surg Radiol Anat. 2004;26:459-461.

6. Duparc F, Bocquet G, Simonet J, Freger P. Anatomical basis of the variable aspects of injuries of the axillary nerve (excluding the terminal branches in the deltoid muscle). Surg Radiol Anat. 1997;19:127-132.

7. Friend J, Francis S, McCulloch J, Ecker J, Breidahl W, McMenamin P. Teres minor innervation in the context of isolated muscle atrophy. Surg Radiol Anat. 2010:32:243-249.

8. Gilroy AM. MacPherson BR. Ross LM. Atlas of Anatomy. 2nd Ed. New York, NY: Thieme; 2012.

9. Hager S, Backus TC, Futterman B, Solounias N, Mihlbachler MC. Posterior subscapular dissection: an improved approach to the brachial plexus for human anatomy students. Ann Anat. 2014;196:88-91.

10. Hashimoto T, Hamada T, Nakamura T, Sasaguri Y, Suzuki K. Myxoid and globular degeneration of nerves in the shoulder joint. Clin Orthop Relat Res. 1995:320:55-64.

11. Moore KL, Dalley AF, Agur AM. Clinically Oriented Anatomy. 7th Ed. Philadelphia, PA: Lippincott, Williams and Wilkins; 2013.

12. Nanjundaiah K, Jayadevaiah SM, Chowdapurkar S. Long head of triceps supplied by axillary nerve. Int J Anat Var. 2012;5:35-37.

13. Neal S, Fields KB. Peripheral nerve entrapment and injury in the upper extremity. Am Fam Physician. 2010;81:147-155.

14. Rezzouk J, Durandeau A, Vital JM, Fabre T. [Long head of the triceps brachii in axillary nerve injury: anatomy and clinical aspects][in French]. Rev Chir Orthop Reparatrice Appar Mot. 2002;88:561-564.

15. Tubbs RS, Oakes WJ, Blount JP, Elton S, Salter G, Grabb PA. Surgical landmarks for the proximal portion of the axillary nerve. J Neurosurg. 2001;95:998-1000. 